\title{
Dispersion mechanisms of nanoclays in a polymer matrix by electron microscopy
}

\author{
M. F. Almeida ${ }^{\text {a,* }}$, A. V. Machado ${ }^{\text {a }}$, J. A. Covas ${ }^{\text {a }}$, N. M. Larocca ${ }^{b}$ and L. A. Pessan ${ }^{\text {b }}$ \\ ${ }^{a}$ I3N-Institute for Nanostructures Nanomodelling and Nanofabrication, University of Minho, 4800- \\ 058 Guimarães, Portugal \\ ${ }^{\mathrm{b}}$ Department of Materials Engineering, Federal University of São Carlos, 13565-905 SP, Brazil \\ *maria.ferreira@dep.uminho.pt
}

Kewords: Nanocomposites; blends; melt compounding; twin screw extruder, dispersion.

\begin{abstract}
.
This work investigates the dispersion of nanoclays in polymer/montmorillonite (MMT)

nanocomposites. Nanocomposites were prepared in a twin screw extruder and the collected samples were analyzed by SEM and TEM. It was possible to study the dispersion of the nanoclays and moreover understand its effect as compatibilizer.
\end{abstract}

\section{Introduction}

Since the preparation of the PA/MMT- based nanocomposites by intercalation mechanism, in 1987 by Okada [1], the research on nanocomposites have been growing. The complex nano domains in polymeric materials can detain a 3D super structure, which results in excellent mechanical and physical properties along with good processability [2-3]. Since nanocomposites are materials with dispersed particles having an average size in the nanometer scale, studies on the dispersion of nanoparticles during melt coumponding have been performed. Even though several studies have provided new insights into the manufacture of nanocomposites in twin screw extruders, fundamental questions related to the evolution of nanoclay dispersion along the screw axis are still to be answered. Thus, the present study aims to investigate the dispersion of the nanoclays in a PA6/PP immiscible blend along the extruder using electronic microscopy.

\section{Material and experimental procedure}

Poliamide 6, PA6, (DOMAMID 27), Polypropylene homopolymer, PP, (MOPLEN - HP502N), a commercial nanoclay, Cloisite 15A (C15A) consisting of MMT modified organically with quaternary alkylammonium salts and PP grafted with maleic anhydride, PP-g-MA (Orevac CA 100) were used.

After drying the PA6 and the nanoclay C 15A under vacuum at $80^{\circ} \mathrm{C}$ during $12-16$ hours, it was tumble-mixed with the other components to a final composition of PA6/PP/C15A of 70/30/5, $\mathrm{w} / \mathrm{w} / \mathrm{w}$, and PA6/PP/PP-g-MA/C15A of 70/30/5/5, w/w/w/w . The blends were processed in a Leistritz LSM 30.34 modular co-rotating intermeshing TSE with a screw diameter of $30 \mathrm{~mm}$ and $\mathrm{L} / \mathrm{D}=29$. The barrel temperature was $230^{\circ} \mathrm{C}$, the screw speed $200 \mathrm{rpm}$ and the feed rate $4 \mathrm{~kg} / \mathrm{h}$. Fig. 1 illustrates the extruder layout, screw geometry and location of the sampling devices. During extrusion, samples were collected along the barrel and quenched in liquid nitrogen for subsequent characterization by SEM and TEM microscopy. Samples characterized by SEM were fractured at low temperature and etched with hot xilene to remove the PP phase. Samples characterized by TEM were sectioned by cryo-ultramicrotomy using a diamond knife of $-45^{\circ} \mathrm{C}$ under liquid nitrogen and then stained with $\mathrm{RuO}_{4}$. 


\section{Results and discussion}

As already know SEM has been established as a convenient technique to analyse blends morphology. Fig. 2 depicts the morphology along the extruder $(\mathrm{L} / \mathrm{D}=8,8$ and 11,9) and at the die of the different prepared blends. As expected, the immiscible PA6/PP blend shows a similar morphology along de axis ( $\mathrm{a}, \mathrm{d}$ and $\mathrm{g}$ ), consisting in large particles of PP dispersed in a PA6 matrix. Blends with nanoclay C15A (b, e and h) and with PP-g-MA and nanoclay (c, f and i) exhibit a different behaviour. As it can be observed in micrographs $h$ and $i$, at the die, the morphology is fine, i.e., a dispersed phase with a very small size is achieved at the die. This difference can be associated with the compatibilization effect of the nanoclay and PP-g-MA. However, micrographs evidenced that compatibilization is more efficient in the presence of PP-g-MA. This efficiency could be explained by the formation of a copolymer between the amine groups of the PA6 and the anhydride groups of PP-g-MA at the interface, which results in stronger interactions than the physical interaction between the nanoclay and polymers. This effect can be better explained by the results illustrated in Fig. 3, TEM micrographic with different magnification (a and b) and (c and d) show that in $\mathrm{PA} / \mathrm{PP} / \mathrm{C} 15$ blend the silicate layers of the nanoclay are mainly located and dispersed in the PA6 phase. However, in PA/PP/PP-g-MA/C15A blend the layers are essentially located at the interface, i. e, between PA6 and PP. As a consequence the compatibilization in this blend is higher due to presence of the copolymer and the higher amount of nanoclays.

\section{Conclusions}

It was shown that both nanoclays and the PP-g-MA act as compatibilizers. However, the compatibilization effect is different, which can be correlated with the dispersion of the nanoclay in polymer matrix.

\section{References}

[1] A. Okada, T. kurauchi, M. Kawasumi et al, Polymer Preparation, 93 (1987).

[2] R. Scaffaro, M.C. Mistretta, F.P. La Mantia, Polymer Degradation and Stability, 93 (2008).

[3] D.W. Schaefer, R.S. Justice, Macromolecules, 40 (2007) 2

The authors are grateful to the FCT (Portuguese Foundation of Science and Technology) for the financial supported.

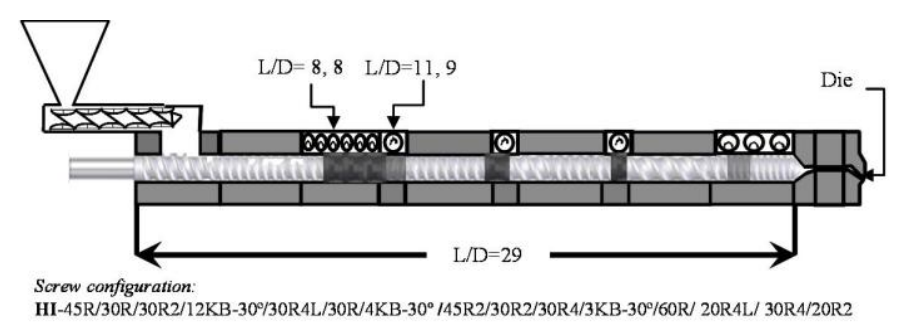

Fig. 1 - Extruder layout and screw configuration

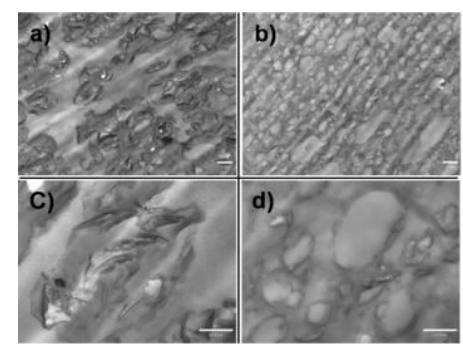

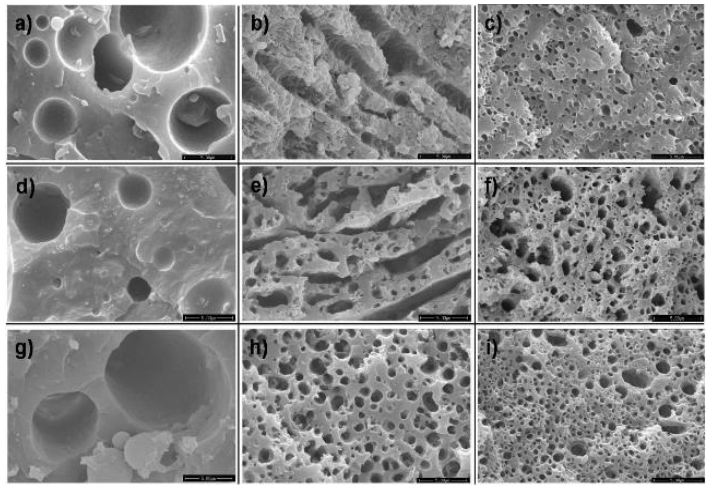

Fig. 2 - SEM micrographics of PA/PP (a, d, g), $\mathrm{PA} / \mathrm{PP} / \mathrm{C} 15 \mathrm{~A}(\mathrm{~b}, \mathrm{e}, \mathrm{h})$ and PA/PP/PP-gMA/C15A (c, f, i) blends at $\mathrm{L} / \mathrm{D}=8,8(\mathrm{a}, \mathrm{b}, \mathrm{c})$, $\mathrm{L} / \mathrm{D}=11,9(\mathrm{~d}, \mathrm{e}, \mathrm{f})$ and die $(\mathrm{g}, \mathrm{h}, \mathrm{i})$

Fig. 3 - TEM micrographics of PA/PP/C15A (a, c) and PA/PP/PP-g-MA/C15A blends (b, d), at $7100(\mathrm{a}, \mathrm{b})$ and $66000(\mathrm{c}, \mathrm{d})$ magnification 